\title{
The UBORA e-infrastructure for open source innovation in medical technology
}

\author{
Carmelo De Maria ${ }^{10000-0002-1368-3571]}$, Licia Di Pietro ${ }^{1}$, Andres Diaz Lantada ${ }^{20000-0003-}$ \\ 3947-6113], Alice Ravizza ${ }^{3}$, Mannan Mridha ${ }^{4}$, Janno Torop ${ }^{5}$, June Madete (60000-0002-1218- $^{6}$ \\ ${ }^{8931]}$, Philippa Makobore ${ }^{7[0000-0002-2501-8392]}$, Arti Ahluwalia 1[0000-0001-5370-6750] \\ ${ }^{1}$ University of Pisa, Pisa, Italy \\ ${ }^{2}$ Universidad Politecnica de Madrid, Madrid, Spain \\ ${ }^{3}$ Politecnico di Torino, Turin, Italy \\ ${ }^{4}$ Royal Institute of Technology, Stockholm, Sweden \\ ${ }^{5}$ University of Tartu, Tartu, Estonia \\ ${ }^{6}$ Kenyatta University, Nairobi, Kenya \\ ${ }^{7}$ Uganda Industrial Research institute, Kampala, Uganda
}

\begin{abstract}
The development of medical devices with open source and collaborative design methodologies has the potential to increase the access to medical technologies, thanks to a feasible reduction of design, management, maintenance, and repairing costs linked to the open access of device blueprints. UBORA is an einfrastructure for the co-design of open source medical devices, which promotes the compliance with internationally recognized quality standards and regulations for safety and efficacy of devices, taking the EN ISO 13485:2016 and the EU MDR 2017/745 as inspiration. UBORA guides the user through a systematic design process, from the identification of clinical needs, of risks class and relevant standards for the device, and provides project management tools, including a repository, finalized to the preparation of the pre-production device dossier. The process is supervised by expert mentors, which ensure that safety and efficacy criteria are fulfilled. The UBORA e-infrastructure is in line with the 2030 Agenda for the Sustainable Development Goals, promoting and strengthening the initiatives of an international community of designers, healthcare providers and policy-makers, toward the reduction of inequalities in the access to medical devices.
\end{abstract}

Keywords: Open source medical devices, e-infrastructure, safety, regulation.

\section{Introduction}

Medical technology is one of the pillars of an effective healthcare system, as recognized by the United Nations Member States in the 2030 Agenda for Sustainable Development Goals (SDGs) [1]. The overall high costs of medical devices (MDs) can be linked to their long life-cycle (design, prototyping, manufacturing, labelling and packaging, provision, installation, operation, maintenance, repair and disposal), during which each step is strictly normed, to guarantee their efficacy and the safety of patients, healthcare providers and bystanders. These costs create a barrier for the accessibility of medical 
technologies: even removing the charge on a single step could not make the difference. For example, the World Health Organization (WHO) estimates that in low income countries more than $80 \%$ of medical equipment is donated, but only $10-30 \%$ of these become operational, given the high operating cost, the lack of personnel and the frequent failures due to harsh environment, extreme climate conditions, humidity, dust and power instability [2-6]. These conditions, not foreseen in the design phase, cause more frequent failures, and determine a higher request for spare parts, which are expensive and difficult to find, making maintenance and repairing as problematic as acquisition [7]. Developing sustainable medical technologies to make healthcare affordable to a larger population, and thus reducing global inequalities, can only be performed taking into account the cultural and socio-economic context in which these will be applied [8].

Compared to the traditional methods of design, the open-source (OS) and collaborative approach can be a possible alternative. By definition, OS means making the design, documentation, source-code, blueprints, ideas and results available for the public. Having the software, electronic and hardware design available under an OS license and in the most suitable file format to study, modify, improve and contribute to the design, potentially leads to very rapid and more reliable innovation [9]. The application of the OS approach to MD design has been proved to offer a unique combination of advantages, such as increasing safety, security, reliability and reducing costs [10]. Until a few years ago, the development of MDs was essentially linked to companies and universities, but recently several examples of open-source MDs (OSMDs) have appeared on the web [11]; however, only some of them have been designed to be compliant with MD legislation [12-14]. It is crucial to ensure the safety and efficacy requirements of medical technology and for this reason the adoption of open resources must follow the standards and the current regulations [15]. To this end, the UBORA e-infrastructure, which merges the OS concepts with the safety and efficacy requirements enforced by the EU Regulation on MDs (MDR) 2017/745, has recently been established [16,17].

\section{The UBORA e-infrastructure}

UBORA provides a structured framework, inspired by the EN ISO 13485:2016 [18] for the identification of needs, risk class, relevant standards, management of computeraided modeling files and preparation of the pre-production device dossier. Each stage is vetted and monitored by expert mentors to ensure that safety criteria are met during the design process. The e-infrastructure includes 3 main sections devoted to the development of OSMDs ("Clinical needs", "Project management", "Resource section"), a part for creating and strengthening a "Community" of developers, mentors and stakeholders, and software tools for the management of the e-infrastructure (Fig.1).

\section{Clinical needs section.}

The clinical needs section is aimed at identifying bioengineering design specifications linked to a particular clinical need. The section provides a unique environment for healthy discussion between patients, healthcare providers and engineers, to ensure that clinical needs are turned into safe and affordable projects. 


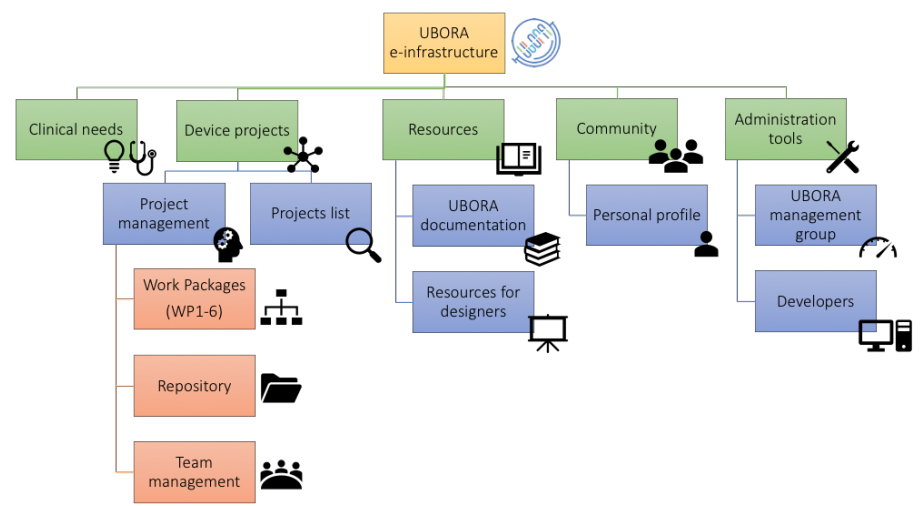

Fig. 1. Overall organization of UBORA e-infrastructure

\section{Project management section.}

This section is a MD oriented project management environment, including a repository, available for free download of project blueprints which have completed the development and compliance assessment phases, under the coordination of expert mentors. This section provides a meta-structure composed of 6 Work Packages (WPs). The medical need and product specification section (WP1) is designed to specify the clinical need and the scope of the MD and to identify the product requirements, as well as clinical areas related to the technology. A list of suggested keywords for indexing the projects are also provided, on the basis of clinical need (e.g. rehabilitation, prevention, etc.), clinical area (e.g. cardiology, neurosurgery, etc.) and technology (e.g. mobilebased, ergonomic support, etc.): this scheme will promote a FAIR data management, ensuring Findability, Accessibility, Interoperability, and Reusability of data [19]. In WP1, the risk class and applicable standards for the specific MD under development are identified. The device classification follows the rules described in Annex VIII of MDR 2017/745. In order to improve usability and minimize human error, the rules are arranged as a decision tree, which guides the designer(s) through a flow of simple (mainly yes/no) questions. Using the same approach, UBORA allows the identification of safety standards, which are usually difficult to identify through keywords in a search engine. WP2 leads the designer in the development of different embodiments/ideas of the same MD, which will be analysed and ranked in a polling section, on the basis of feasibility, performance, usability and safety aspects. In WP3, focused on design and prototyping, the designers collaboratively work on the MD idea selected in WP2: the project management system suggests the organization in subsections related to mechanical components, electronics/firmware, software, and their integration. Once WP3 is concluded, WP4 leads the developers in the testing phase to demonstrate the compliance with relevant standards. WP5 is aimed at completing the technical documentation of the device in order to publish the project for downloading, and provides a lean business model canvas [20], customized for OSMDs, to help the potential transfer of the technology to industry. Finally, WP6 guides the developers in creating promotional videos and brochures, toward a possible crowdfunding campaign. 


\section{Resource section.}

The resource section contains a selected teaching/learning material on biomedical engineering, as well as the documentation and video tutorials to use and further develop the e-infrastructure, whose source code will be released as open source by the end of the UBORA project. In particular UBORA is built on Microsoft's ASP.NET Core web framework, using $\mathrm{C} \#$ as programming language for the back-end, while the front- end is a multi-page application based on JavaScript, rendered on the server side and then sent to the client (browser).

UBORA was officially launched to the public during the conference of Collaborative Biomedical Engineering for Open Source Medical Technologies and the successive UBORA Design School 2018, held in Pisa from the $1^{\text {st }}$ to the $7^{\text {th }}$ September. To date the e-infrastructure, available at https://platform.ubora-biomedical.org, has 485 users and 286 projects, at different stage of development (Fig. 2), both on high-tech innovative MDs, such as a wearable device for the breast self-examination [14] or 3D printed splints, and smart accessories to existing MDs, as a timer for the AMBU bag.
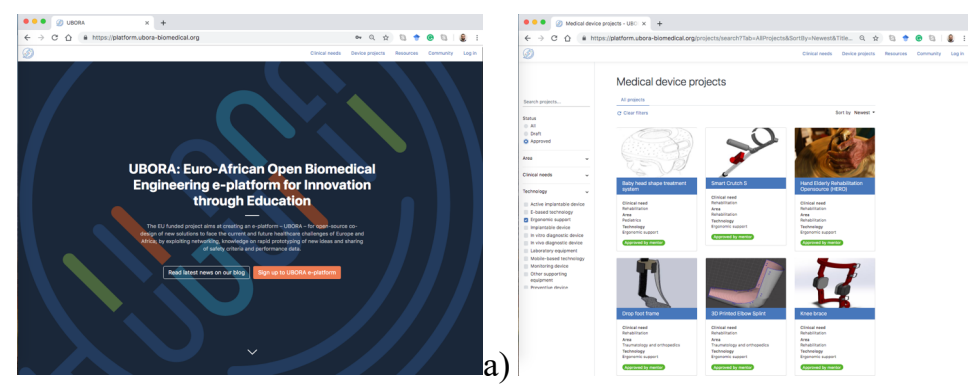

b)

Fig. 2. UBORA e-infrastructure. Landing page (a) and some medical device projects, selected through specific keywords (b)

\section{Conclusion}

The UBORA e-infrastructure provides a framework to develop ISO compliant MDs starting from clinical needs by sharing idea, blueprints and data. If properly implemented, these devices can be considered in line with the MDR 2017/745 from the design point of view. In a nutshell, its final aim is to promote well-being for all, increasing access to MDs and moving towards health equity in accordance with the UN SDGs.

\section{Acknowledgements}

This project has received funding from the European Union's Horizon 2020 research and innovation programme under grant agreement $\mathrm{N}^{\circ}$ 731053. The authors acknowledge all students and mentors who daily animate the UBORA e-infrastructure. 


\section{Conflict of interest}

The authors have no conflict of interest to disclose.

\section{References}

1. Sustainable Development Goals. https://www.un.org/sustainabledevelopment/sustainabledevelopment-goals/ last access: May 2019]

2. World Health Organization. Medical devices: managing the mismatch: an outcome of the priority medical devices project. World Health Organization (2010).

3. Iadanza, E., and Dyro, J., "Clinical engineering handbook". Elsevier (2004).

4. World Health Organization. Barriers to innovation in the field of medical devices. Background paper 6. Geneva, World Health Organization (2010).

5. Malkin, R. A. "Design of health care technologies for the developing world." Annu. Rev. Biomed. Eng. 9: 567-587 (2007).

6. Lustick, D. R., and Zaman, M. H., "Biomedical engineering education and practice challenges and opportunities in improving health in developing countries." Science and Innovation Policy, 2011 Atlanta Conference on. IEEE (2011).

7. Malkin, R. A. "Barriers for medical devices for the developing world". Expert review of medical devices, 4(6), 759-763 (2007).

8. Douglas, T. S., "Biomedical engineering education in developing countries: research synthesis." EMBC. IEEE (2011).

9. Perens, B., "The open source definition." Open sources: voices from the open source revolution 1: 171-188 (1999).

10. Williams, A., Gibb, A., and Weekly, D., "Research with a hacker ethos: what DIY means for tangible interaction research". Interactions, 19(2), 14-19 (2012).

11. Niezen, G., Eslambolchilar, P., and Thimbleby H., "Open-source hardware for medical devices". BMJ innovations bmjinnov-2015 (2016).

12. Gamma Cardio Soft S.r.l. http://www.gammacardiosoft.it/openecg/ last access: May 2019.

13. Ferretti, J., Di Pietro, L., \& De Maria, C., "Open-source automated external defibrillator. HardwareX, 2, 61-70 (2017).

14. Arcarisi, L., Di Pietro, L., Carbonaro, N., Tognetti, A., Ahluwalia, A., De Maria, C. "Palpreast-A New Wearable Device for Breast Self-Examination". Appl. Sci.9,381 (2019).

15. De Maria, C., Mazzei, D., and Ahluwalia, A., "Improving African health care through open source Biomedical Engineering." International Journal on Advances in Life Sciences 7.1:1019 (2015).

16. UBORA: Euro-Africa Open Biomedical Engineering Innovation e-platform for Innovation through Education. https://platform.ubora-biomedical.org/ last access: May 2019.

17. European Medical Device Regulation 2017/745. https://eur-lex.europa.eu/legal-content/EN/TXT/PDF/?uri=OJ:L:2017:117:FULL\&from=EN last access: May 2019.

18. International Organization of Standardization EN ISO 13485:2016 Medical devices - Quality management system- Requirements for regulatory purpose certification. https://www.iso.org/standard/59752.html last access: May 2019.

19. Wilkinson, M. D., Dumontier, M., Aalbersberg, I. J., Appleton, G., Axton, M., Baak, A., ... \& Bouwman, J. The FAIR Guiding Principles for scientific data management and stewardship. Scientific data, 3 (2016).

20. Trimi, S., \& Berbegal-Mirabent, J. "Business model innovation in entrepreneurship". International Entrepreneurship and Management Journal, 8(4), 449-465 (2012). 